\title{
Glycemic Control in Diabetic Patients in Saudi Arabia: The Role of Knowledge and Self-Management - A Cross-Sectional Study
}

\author{
Saad M. AlShareef, MD, MHPE ${ }^{1}$, Abdullah A. AlWabel, MD², Mujahed A. AlKhathlan, MD', \\ Ahmed A. AlKhazi ${ }^{1}$, Abdulaziz K. AlMaarik ${ }^{1}$, Abdullah M. AlGarni ${ }^{1}$, \\ Abdullah Saeed AlGhamdi ${ }^{1} \&$ Mohammed D. AlMutairi ${ }^{1}$ \\ ${ }^{1}$ Department of Medicine, College of Medicine, Al Imam Mohammad Ibn Saud Islamic University (IMSIU), \\ Riyadh, Saudi Arabia \\ ${ }^{2}$ Internal Medicine Department, King Saud Medical City, Riyadh, Saudi Arabia \\ ${ }^{3}$ King Abdullah Scholarship Program, Ministry of Education, Riyadh, Saudi Arabia \\ Correspondence: Saad M. AlShareef, Othman Ibn Afan Road, Exit 5, PO Box 7544, Riyadh, Saudi Arabia. Tel: \\ 966-112-037-109. E-mail: drsaad321@hotmail.com
}

Received: August 28, 2017 Accepted: September 25, 2017 Online Published: September 27, 2017

doi:10.5539/gjhs.v9n12p25 URL: https://oi.org/10.5539/gjhs.v9n12p25

\begin{abstract}
Introduction: Diabetes mellitus (DM) is serious healthcare concern in Saudi Arabia, with the disease's prevalence in the country being one of the highest worldwide. This study examines various factors which affect outcomes of patients with DM; namely, medication adherence, diabetes knowledge, self-management behaviours, and glycemic control.
\end{abstract}

Methods: This is a cross-sectional survey-based study. Participants were patients with a DM diagnosis at King Saud Medical City in Riyadh, Saudi Arabia.

Results: Positive associations were found between medication adherence and diabetes knowledge; self-management behaviours (glucose management and healthcare use) and diabetes knowledge; self-management behaviours (dietary control) and fasting blood glucose levels; and age and blood glucose levels (both fasting and $\mathrm{HgA} 1 \mathrm{c})$. No associations were found between diabetes knowledge and glycemic control; or between self-management behaviours and $\mathrm{HgA} 1 \mathrm{c}$ levels.

Conclusion: Having good knowledge of diabetes is associated with medication adherence and healthcare self-management. Healthcare practitioners should consider educating DM patients an integral part of the treatment process.

Keywords: diabetes mellitus, glycemic control, patient knowledge, self-management

\section{Introduction}

Diabetes mellitus (DM) is a fast-growing healthcare problem worldwide, which is now reaching epidemic proportions in some countries. In 2014, it was estimated that almost 422 million people suffered from diabetes, with a global prevalence of $8.5 \%$ (World Health Organization [WHO], 2016). Saudi Arabia is among the 10 countries with highest prevalence of DM worldwide (23.9\%; Aguiree et al., 2013). Many diabetes patients suffer from complications that have a significant impact on their quality of life. In 2012, an estimated 1.5 million deaths were directly caused by diabetes, and higher-than-optimal blood glucose was responsible for an additional 2.2 million deaths due to increased risks of cardiovascular disease and other diseases. This equates to a total of 3.7 million deaths related to blood glucose levels (WHO, 2016). The WHO expects that diabetes will be the seventh leading cause of death by 2030 . This underlines the need to examine the factors which improve outcomes for patients with DM, and in particular, the factors which contribute to improved glycemic control.

Research has shown that medication adherence significantly contributes to improved blood glucose (HbA1c) levels (Rhee et al., 2005). Non-compliance with treatment plans and poor medication adherence is a significant problem for diabetic patients in Saudi Arabia. One study found that therapeutic non-compliance among a sample of diabetic patients in the Al Hasa region of Saudi Arabia was at 67.9\% (Khan et al., 2012), with various factors such as poor education and urban living being associated with higher levels of non-compliance. An investigation 
into the factors which contribute to poor medication adherence in Saudi Arabia is therefore warranted.

Compliance can also be described as the degree to which patients take an active role in their healthcare self-management. A systematic review (Norris, Engelgau, \& Narayan, 2001) found that in studies with a follow up of less than 6 months, self-management training had a positive effect on diabetes knowledge, frequency and accuracy of blood glucose self-monitoring, self-reported dietary habits, and glycemic control. The effect of self-management training on glycemic control is again supported by a study which found that self-management training significantly decreased GHb levels in adults with Type 2 diabetes at immediate follow-up, 1-3 month follow up, and $\geq 4$ month follow up when compared to a control group (Norris, Lau, Smith, Schmid, \& Engelgau, 2002).

These findings are in line with the self-determination theory (SDT) of health behaviour change, which posits that the psychological needs of competence and autonomy each contribute to enhanced self-motivation. Changes in perceptions of competence and autonomy have indeed been shown to correlate with changes in glycemic control. Autonomous motivation (behaviour that is conducted through one's own choice rather than through coercion) and perceived competence (the perception that one can control outcomes related to their illness) have both been found to contribute to improved glycemic control (Williams, McGregor, Zeldman, Freedman, \& Deci, 2004). This suggests that DM patients may benefit from practitioner-driven education that would inform them of their own role in controlling their disease outcomes, as this would increase their autonomous motivation to, and perceived competence in, engaging in self-management behaviours. Indeed, when healthcare providers are more supportive of patient autonomy, patients have higher levels of autonomous motivation and perceived competence, which in turn leads to improvements in their blood glucose levels (Heisler, Smith, Hayward, Krein, \& Kerr, 2003; Williams, Freedman, \& Deci, 1998). It is therefore important to consider self-management behaviours as a key determinant of DM patient outcomes.

It is evident from the above examples that practitioner-supported autonomy can increase a patient's perceived competence, and encourage self-management behaviours. For this reason, we also consider patients' diabetes knowledge to play an important role in their healthcare behaviour and outcomes. Al-Qazaz et al. (2011) found an association between patients' diabetes knowledge and medication adherence, with patients who were more knowledgeable about their disease adhering more closely to their treatment regimen than those with poor knowledge. As expected, better diabetes knowledge and medication adherence both correlated with better glycemic control. Furthermore, increases in diabetes knowledge after patients had received diabetes self-management education (DSME) have been shown to predict the achievement of target A1c levels (Berikai et al., 2007). This is suggestive of a link between self-management behaviours and diabetes knowledge with respect to their effect on glycemic control.

While these findings are compelling, it is important to examine the role of medication adherence, self-management behaviours and diabetes knowledge in glycemic control within a Saudi context. Based on a review of the literature, we present the following hypotheses:

1). There is a positive association between diabetes knowledge and medication adherence.

2). There is a positive association between diabetes knowledge and self-management behaviours.

3). There is a positive association between diabetes knowledge and glycemic control.

4). There is a positive association between self-management behaviours and glycemic control.

\section{Methodology}

\subsection{Design}

This is a cross-sectional study consisting of interview-based surveys, conducted between February and April 2017. Data were collected in diabetic clinics at King Saud Medical City in Riyadh, Saudi Arabia. Ethical approval was obtained from the Institutional Review Board of Al-Imam Muhammad Ibn Saud Islamic University. This study conforms to the principles of the Helsinki Declaration.

\subsection{Participants}

All participants had a diagnosed case of DM, and had been receiving treatment for at least 6 months prior to enrolment. Patients were excluded if they were not taking medication for diabetes, if they had gestational diabetes, or if they were diagnosed with a psychiatric illness.

512 total participants took part in this study. Participants were aged between 18 and 83 years old $(M=52.45, S D$ $=13.22$ ). $11.3 \%$ had a diagnosis of Type 1 diabetes, with $88.7 \%$ having Type 2 . Mean time since diagnosis was 
11.68 years $(S D=9.57) .50 .6 \%$ of patients used oral hypoglycemic agents as their only form of treatment, while $42.7 \%$ used insulin only, and $6.7 \%$ used both. See Table 1 for frequencies and Table 2 for descriptive statistics.

Informed consent was obtained from all participants before commencement in this study.

Table 1. Frequencies

\begin{tabular}{|c|c|c|c|c|}
\hline \multirow[t]{2}{*}{ Gender } & \multicolumn{2}{|l|}{ Male } & \multicolumn{2}{|l|}{ Female } \\
\hline & \multicolumn{2}{|l|}{$356(69.4 \%)$} & \multicolumn{2}{|l|}{$156(30.4 \%)$} \\
\hline \multirow{2}{*}{ Marital status } & Married & Single & Divorced & Widowed \\
\hline & $452(88.1 \%)$ & $39(7.6 \%)$ & $2(.4 \%)$ & $1(.2 \%)$ \\
\hline \multirow[t]{2}{*}{ DM Type } & Type 1 & & Type 2 & \\
\hline & $58(11.3 \%)$ & & $454(88.7 \%)$ & \\
\hline \multirow[t]{3}{*}{ DM Management } & Oral Hypoglycemic & Insulin Only & & Both \\
\hline & Agents Only & & & \\
\hline & $258(50.6 \%)$ & $218(42.7 \%)$ & & $34(6.7 \%)$ \\
\hline \multirow[t]{2}{*}{ Education Level } & Illiterate & Primary & Secondary & Third Level \\
\hline & $99(21.1 \%)$ & $90(19.2 \%)$ & $173(36.9 \%)$ & $107(22.8 \%)$ \\
\hline
\end{tabular}

Note. $\mathrm{DM}=$ Diabetes Mellitus.

Table 2. Descriptive statistics

\begin{tabular}{lll}
\hline Variable & M & SD \\
\hline Age & 52.45 & 13.22 \\
Weight $(\mathrm{kg})$ & 78.82 & 18.89 \\
Height $(\mathrm{cm})$ & 166.71 & 9.29 \\
BMI & 28.95 & 7.24 \\
Years since diagnosis & 11.68 & 9.57 \\
Glucose (HGA1C) & 8.71 & 2.43 \\
Glucose (fasting) & 10.74 & 4.73 \\
\hline
\end{tabular}

Note. $B M I=$ Body Mass Index; HGA1C = Hemoglobin A1C

\subsection{Measures}

To measure medication adherence, the 8-Item Morisky Medication Adherence Scale (MMAS-8) was used. This scale consists of seven yes/no statements, and one Likert-scale question. A high score on this scale indicates poor medication adherence. This scale has been shown to demonstrate both concurrent and predictive validity (Morisky, Green, \& Levine, 1986).

The Diabetes Self-Management Questionnaire (DSMQ) was used to evaluate self-care activities. This scale has been shown to have good internal consistency, and to correlate significantly with HbA1c levels (Schmitt et al., 2013). This scale consists of a series of 4-point Likert-scale statements, with a high score indicating a high level of autonomy in the patient's disease management. This scale defines different aspects of self-management through the following subscales: 'Glucose Management', 'Dietary Control', 'Physical Activity', and 'Health-Care Use'.

The revised Diabetes Knowledge Test (DKT2) was used to measure patient's knowledge of diabetes. The test consists of a 14-item test of general diabetes knowledge, and a 9-item test for patients who use insulin as part of their treatment. For the purpose of this study, only the 14-item general test was administered. The DKT2 has been shown to have good internal consistency and validity (Fitzgerald et al., 2016).

Participants also answered questions about their demographics and treatment. Current blood glucose levels were obtained from patients' medical files. 


\subsection{Analysis}

Data were analysed using correlational and regression analyses, as well as independent samples t-tests. Analyses were performed using IBM SPSS Statistics package, version 24.

\section{Results}

\subsection{Preliminary Analysis}

Significant correlations were found between DKT2 score and MMAS8 score, $r(488)=-.214, p<.001$. Significant correlations were also found between DKT2 score and the DSMQ, $r(487)=.215, p<.001$; DKT2 and the DSMQ Glucose Management Subscale, $r(485)=.184, p<.001$; DKT2 and the DSMQ Dietary Control Subscale, $r(486)$ $=.14, p<.01$; and the DKT2 and the DSMQ Healthcare Use Subscale, $r(487)=.199, p<.001$

Apart from HgA1c levels, only two variables correlated with fasting glucose levels: age, $r(490)=-.167, p<.001$; and the DSMQ Dietary Control Subscale, $r(485)=-.105, p<.05$.

Apart from fasting glucose levels, the only variable to correlate with $\mathrm{HgAlc}$ levels was age, $r(490)=-.246, p$ $<.001$. See Table 3 for a summary of correlations.

\subsection{Diabetes Knowledge and Medication Adherence}

A regression model with MMAS8 total score and the MMAS8 Likert-scale variable as explanatory variables fit the diabetes knowledge data well, $R^{2}=.055, F(2,487)=14.183, p<.001$, with DKT2 scores being significantly explained by the MMAS8.

Table 3. Correlations

\begin{tabular}{|c|c|c|c|c|c|c|c|c|c|c|c|}
\hline & 1 & 2 & 3 & 4 & 5 & 6 & 7 & 8 & 9 & 10 & 11 \\
\hline \multicolumn{12}{|l|}{ 1. Age } \\
\hline 2. Years since diagnosis & $.262 * *$ & & & & & & & & & & \\
\hline 3. DSMQ Total & .018 & .051 & & & & & & & & & \\
\hline 4. DSMQ Glucose & .04 & $.102 *$ & $.757 * *$ & & & & & & & & \\
\hline Management & & & & & & & & & & & \\
\hline 5. DSMQ Dietary Control & $.103^{*}$ & .063 & $.723 * *$ & $.42 * *$ & & & & & & & \\
\hline $\begin{array}{l}\text { 6. DSMQ Physical } \\
\text { Activity }\end{array}$ & $-.114 *$ & -.071 & $.599 * *$ & $.236^{* *}$ & $.294 * *$ & & & & & & \\
\hline 7. DSMQ Healthcare Use & -.027 & .056 & $.645 * *$ & $.37 * *$ & $.292 * *$ & $.189 * *$ & & & & & \\
\hline 8. DKT2 score & -.033 & .036 & $.215^{* *}$ & $.184^{* *}$ & $.14^{* *}$ & .078 & $.199^{* *}$ & & & & \\
\hline 9. MMAS8 & .019 & -.042 & $-.41 * *$ & $-.432 * *$ & $-.237 * *$ & $-.169 * *$ & $-.315^{* *}$ & $-.214 * *$ & & & \\
\hline 10. MMAS8 Likert scale & .048 & -.029 & $-.26 * *$ & $-.266^{* *}$ & $-.115^{* *}$ & $-.149 * *$ & $-.221 * *$ & $-191 * *$ & $.498 * *$ & & \\
\hline 11. $\mathrm{HgAlc}$ & $-.246 * *$ & .042 & .044 & -.026 & -.08 & -.014 & .031 & .046 & -.042 & -.08 & \\
\hline 12. Glucose fasting & $-.167 * *$ & .058 & .075 & -.047 & $-.105^{*}$ & -.084 & .074 & .042 & -.045 & -.056 & $.702 * *$ \\
\hline
\end{tabular}

$* p<.05$

$* * p<.01$

Total score, $\beta=-.157, t(486)=-3.057, p<.01$; and the MMAS8 Likert-scale, $\beta=-.112, t(486)=-2.187, p<.05$.

\subsection{Diabetes Knowledge and Self-Management}

A regression model with the DSMQ Glucose Management Subscale, the DSMQ Dietary Control Subscale and the DSMQ Healthcare Use Subscale as explanatory variables fit the DKT2 score data well, $R^{2}=.057, F(3,475)=9.62$, $p<.001$, with DKT2 scores being significantly explained by the DSMQ Glucose Management Subscale, $\beta=.106$, 
$t(474)=2.069, p<.05$; and the DSMQ Healthcare Use Subscale, $\beta=.152, t(474)=3.109, p<.01$; but not by the DSMQ Dietary Control Subscale.

\subsection{Diabetes Knowledge and Glycemic Control}

No significant correlation was found between DKT2 scores and either HgA1c levels or fasting glucose levels. A median split was performed on DKT2 scores, using 5 as the median. Those who scored 5 or below were labelled poor performers, with those scoring above 5 being labelled high performers. Independent samples t-tests showed no significant differences between high and low performers in HgA1c levels, $t(472)=-.126, p=.9$; or fasting glucose levels, $t(472)=.227, p=.82$.

Factorial ANOVAs were also conducted to determine if there was any interaction between type of DM and diabetes knowledge for glucose levels. While a main effect was found for DM type on $\mathrm{HgAlc}$ levels, $F(1,470)=$ $16.231, p<.001, \eta_{\text {partial }}^{2}=.033$, and on fasting glucose levels, $F(1,470)=6.622, p=.01, \eta_{\text {partial }}^{2}=.014$, there was no main effect found for diabetes knowledge, and no significant interaction.

Post-hoc independent samples t-tests confirmed the difference in HgAlc levels between Type $1(M=10.067, S D=$ $2.572)$ and Type 2 patients $(M=8.535, S D=2.361), t(490)=4.492, p<.001$; and the difference in fasting glucose levels between Type $1(M=12.527, S D=5.53)$ and Type 2 patients $(M=10.514, S D=4.574), t(63.641)=2.59, p$ $=.012$. However, because of the difference in sample sizes across the two groups, statistical power is quite low. For this reason, the data have not been split to consider regression models for both groups. Additional independent samples t-tests for MMAS8, DKT2 and DSMQ scores revealed no significant differences between the two patient groups.

\subsection{Self-Management and Glycemic Control}

Based on preliminary correlational results, a regression model was formed with the DSMQ Dietary Control Subscale and age as explanatory variables, and fasting glucose levels as the outcome variable. The model fit the data well, $R^{2}=.036, F(2,484)=9.036, p<.001$, with glucose readings being significantly explained by both the DSMQ Dietary Subscale, $\beta=-.091, t(483)=-2.033, p<.05$; and age, $\beta=-.158, t(483)=-3.535, p<.001$.

The same model also fit the data for $\mathrm{HgA} 1 \mathrm{c}$ readings well, $R^{2}=.064, F(2,484)=16.418, p<.001$. However, age was the only significant explanatory variable, $\beta=-.24, t(483)=-5.435, p<.001$.

\subsection{Additional Observations}

Independent samples t-tests revealed that patients who use oral hypoglycemic agents exclusively reported lower HgAlc levels $(M=8.411, S D=2.344)$ than those who do not $(M=9.022, S D=2.486), t(488)=2.801, p<.01$; and also reported lower glucose fasting levels $(M=10.253, S D=4.459)$ than those who $\operatorname{don} \operatorname{not}(M=11.27, S D=4.948)$, $t(481.07)=2.378, p<.05$.

In addition, patients who use insulin exclusively as a treatment option reported higher HgAlc levels $(M=9.102$, $S D=2.488)$ than those who do not $(M=8.417, S D=2.347), t(489)=-3.123, p<.01$; and also reported higher glucose fasting levels $(M=11.392, S D=4.978)$ than those who do not $(M=10.263, S D=4.479), t(425.62)=$ $-2.596, p<.05$.

\section{Discussion}

We set out to examine the interplay between medication adherence, diabetes knowledge, self-management behaviours, and glycemic control in patients with a diagnosis of diabetes mellitus (DM). Our findings suggest a positive association between medication adherence and patient knowledge of diabetes. This provides further support to research which found that patients who were more knowledgeable about their disease adhered more closely to their treatment regimen than those with poor knowledge (Al-Qazaz et al. 2011). This is in line with self-determination theory (SDT) which considers perceived competency as an integral factor in determining whether or not patients engage in positive health behaviours that would improve their disease outcomes.

Further complimenting SDT is the positive association found between diabetes knowledge and both glucose management and healthcare use behaviours. Our findings suggest that the more knowledge a patient has about his/her disease, the more likely he/she is to engage with the healthcare process by attending regular appointments with their healthcare provider, and manage their glucose levels by taking their prescribed medication and monitoring their blood glucose levels regularly. While there was a positive correlation between the dietary control subscale and diabetes knowledge, dietary control did not emerge as a significant explanatory variable in the regression model. This is perhaps due to the controlling effect of the other subscales in this model. The lack of association between diabetes knowledge and the physical activity subscale may be due to the fact that the DKT2 only contains one question related to physical activity, and therefore may not be a robust enough measure of a 
patient's knowledge of how physical activity can improve outcomes for diabetic patients.

Our hypothesis that good diabetes knowledge would be associated with good glycemic control was not supported. This is true for both $\mathrm{HgAlc}$ and fasting glucose levels. This is in contrast with previous research (Al-Qazaz et al., 2011; Berikai et al., 2007). A key difference between the present study and previous studies (Berikai et al., 2007) is that they examined blood glucose levels at two time points: before and after receiving diabetes self-management education. They therefore examine the effect that a gain in knowledge has on glycemic control across time. The lack of a significant association here does not imply that such educational initiatives lack merit, but suggests that an increase in knowledge may be a more compelling predictor of glycemic control than knowledge at a single time point.

Our hypothesis that there would be an association between self-management behaviours and glycemic control was partially supported for fasting glucose levels by a regression model including dietary self-management behaviours and age. Scoring high in dietary control behaviours was associated with lower fasting glucose levels, but not with $\mathrm{HgAlc}$ levels. It stands to reason that engaging in dietary control behaviours would result in lower blood glucose levels at a particular time point, while not affecting $\mathrm{HgAlc}$ levels which are average blood glucose levels across time. It would be interesting to examine this longitudinally, as this would allow for the examination of changes in dietary control behaviours across time. Such an approach may unearth an association with HgA1c levels.

The lack of association between other self-management behaviours and blood glucose levels was more surprising. A key limitation of this study is that participants' behaviour was self-reported and not measured at different time points. Cross-referencing participants' self-reports with those of their caregivers or close family members may give more insight into each patient's relationship with their illness. The fact that age emerged as a significant explanatory variable for both blood glucose readings suggests that older age is associated with better glycemic control. As older patients have generally been living with the disease for longer than younger patients, their treatment plan may have already evolved from the trial and error period, which may result in better glycemic control. It may be interesting for future studies to examine the reasons behind this effect.

Another limitation of the present study is that the sample contains significantly more patients with Type 2 diabetes than Type 1 diabetes. While Type 1 patients had significantly higher glucose levels than Type 2 patients in our sample, the difference in group sizes leads to limited statistical power. Future research with a more balanced sample could examine the regression models presented in sections 3.2,3.3 and 3.5 to see if they are a good fit for both patient groups.

\section{Conclusion}

It is important for healthcare practitioners to ensure DM patients are knowledgeable about their disease. This will allow patients to take a more active role in their healthcare self-management, and may encourage behaviours such as dietary control which could improve their glycemic control. Patients are also more likely to adhere to their prescribed medication plan if they know how taking their medication affects their healthcare outcomes. While it is not clear whether there is an association between diabetes knowledge and glycemic control, it is essential that patients are encouraged to engage in self-management behaviours such as physical activity, glucose management, dietary control, and healthcare use, so that they feel actively engaged in the healthcare process. This process begins with the healthcare practitioner educating the patient on the facts of their illness, their treatment, and optimal outcomes.

\section{Acknowledgements}

The authors would like to thank all the patients that participated in this study. The authors received no financial support for this research and authorship of this article, and there are no conflicts of interest.

\section{Competing Interests Statement}

The authors declare that there are no competing or potential conflicts of interest.

\section{References}

Aguiree, F., Brown, A., Cho, N. H., Dahlquist, G., Dodd, S., Dunning T., ...Whiting, D. (2013). IDF diabetes atlas (6th ed.). Basel, Switzerland: International Diabetes Federation.

Al-Qazaz, H. K., Sulaiman, S. A., Hassali, M. A., Shafie, A. A., Sundram, S., Al-Nuri, R., \& Saleem, F. (2011). Diabetes knowledge, medication adherence and glycemic control among patients with type 2 diabetes. International Journal of Clinical Pharmacy, 33(6), 1028-1035. https://doi.org/10.1007/s11096-011-9582-2

Berikai, P., Meyer, P. M., Kazlauskaite, R., Savoy, B., Kozik, K., \& Fogelfeld, L. (2007). Gain in patients' 
knowledge of diabetes management targets is associated with better glycemic control. Diabetes Care, 30(6), 1587-1589. https://doi.org/10.2337/dc06-2026

Fitzgerald, J. T., Funnell, M. M., Anderson, R. M., Nwankwo, R., Stansfield, R. B., \& Piatt, G. A. (2016). Validation of the revised brief Diabetes Knowledge Test (DKT2). Diabetes Education, 42(2), 178-187. https://doi.org/10.1177/0145721715624968

Heisler, M., Smith, D. M., Hayward, R. A., Krein, S. L., \& Kerr, E. A. (2003). How well do patients' assessments of their diabetes self-management correlate with actual glycemic control and receipt of recommended diabetes services? Diabetes Care, 26(3), 738-743.

Khan, A. R., Lateef, Z. N. A. A., Al Aithan, M. A., Bu-Khamseen, M. A., Al Ibrahim, I., \& Khan, S. A. (2012). Factors contributing to non-compliance among diabetics attending primary health centers in the Al Hasa district of Saudi Arabia. Journal of Family and Community Medicine, 19(1), 26. https://doi.org/10.4103/2230-8229.94008

Morisky, D.E., Green, L.W., \& Levine, D.M. (1986). Concurrent and predictive validity of a self-reported measure of medication adherence. Medical Care, 24(1):67-74.

Norris, S. L., Engelgau, M. M., \& Narayan, K. V. (2001). Effectiveness of self-management training in type 2 diabetes. Diabetes Care, 24(3), 561-587.

Norris, S. L., Lau, J., Smith, S. J., Schmid, C. H., \& Engelgau, M. M. (2002). Self-management education for adults with type 2 diabetes. Diabetes Care, 25(7), 1159-1171.

Rhee, M. K., Slocum, W., Ziemer, D. C., Culler, S. D., Cook, C. B., El-Kebbi, I. M., ... Phillips, L. S. (2005). Patient adherence improves glycemic control. Diabetes Education, 31(2), 240-250. https://doi.org/10.1177/0145721705274927

Schmitt, A., Gahr, A., Hermanns, N., Kulzer, B., Huber, J., \& Haak, T. (2013). The Diabetes Self-Management Questionnaire (DSMQ): Development and evaluation of an instrument to assess diabetes self-care activities associated with glycaemic control. Health and Quality of Life Outcomes, 11(1), 138. https://doi.org/10.1186/1477-7525-11-138

Williams, G. C., Freedman, Z. R., \& Deci, E. L. (1998). Supporting autonomy to motivate patients with diabetes for glucose control. Diabetes Care, 21(10), 1644-1651.

Williams, G. C., McGregor, H. A., Zeldman, A., Freedman, Z. R., \& Deci, E. L. (2004). Testing a self-determination theory process model for promoting glycemic control through diabetes self-management. Health Psychology, 23(1), 58. https://doi.org/10.1037/0278-6133.23.1.58

World Health Organization. (2016). WHO mortality database. Geneva, Switzerland: World Health Organization. Retrieved from: http://apps.who.int/healthinfo/statistics/mortality/causeofdeath_query/

\section{Copyrights}

Copyright for this article is retained by the author(s), with first publication rights granted to the journal.

This is an open-access article distributed under the terms and conditions of the Creative Commons Attribution license (http://creativecommons.org/licenses/by/4.0/). 